\title{
Terror and Tears in the Labour suit: The Prevalence and Forms of patient Abuse by Health workers during Childbirth in Uganda
}

\author{
Article by Kasule Aaron ${ }^{1}$, Jerome K. Kabakyenga ${ }^{2}$ \\ ${ }^{1}$ Lecture department of public health Kampala international university, Uganda \\ ${ }^{2}$ Professor of Public health Mbarara University of science and technology, Uganda \\ Email: khaaron2000@yahoo.com
}

\begin{abstract}
Background: Among the factors contributing to the high maternal morbidity and mortality in Uganda is the proportion of pregnant women who do not deliver under skilled supervision. There is paucity of data concerning the magnitude of patient abuse by health workers which is a main factor preventing facility delivery. This study aimed to determine the magnitude of abuse and the forms of abuse the patients in Bushenyi district in Uganda. Methods: In this cross sectional survey, 374 women who had just delivery in the health facilities were interviewed to identify any form of abuse treatment. The independent predictors of patient abuse were analysed at bivariate and multivariate level using binary logistic regression analysis. Results: The prevalence of patient abuse among the respondents was found to be 41.1\% ( $P=0.013)$. The commonest form of abuse reported was patient neglect $31.9 \%$ ( $p=$ 0.000), verbal abuse (29.3\%) and bribery (15.1\%). The associated with patient abuse were; patient age 15-25years, prime gravidity and low Health center facility. Conclusions: The prevalence of patient abuses during childbirth very high and detrimental the health system. Close supervision midwives especially in the lower level facilities, as well as keeping the company of the relatives or friends to especially the younger the patients around the labour suite, Community sensitisation and empowerment through health education are suggested.
\end{abstract}

Keywords: Patient abuse, childbirth, South Western Uganda

\section{Introduction}

Each year, over 850 women die during child birth [1-4] and ninety percent of these deaths occur in how income countries $[2,3]$. In the recent years, concerted efforts towards maternal survival have made significant stride in reducing maternal mortality due to preventable cause $[1,2,5]$ in the developed world with the remaining dismal, particularly due to un avoidable circumstances $[3,6]$. Despite the in comprehendible contribution to the magnitude of avoidable maternal mortality and morbidity, patient neglect and abuse has historically been a forgotten phenomenon. Unfortunately the relative oversight put to it is multilevel in nature including the policy making level, managerial level and at the implementation level of the health system $[2,6]$. Furthermore, the safe motherhood initiative launched over thirty years ago doesn't pay attention to maternal abuse and neglect $[7,8]$. The relative neglect of this health system impairing phenomenon and the failure to implement interventions against it are seen most significantly in sub-Saharan Africa [9]. Here the presumably associated maternal mortality rates are highest and uptake of delivery services lowest $[4,10]$.

In Uganda the low utilization of health facility delivery service and the resultant maternal mortality and morbidity have been closely linked to the ethical behavior of the health workers and human right issues encountered during practice $[5,6,11]$. It is surprising that, over time, patient neglect and abuse have not been targeted in programs aimed at attending to the health of the pregnant woman in this country. It has been proposed that the reasons behind such an atmosphere lie in myths that it's impossible to have a successful delivery without the use of excessively strong language and actions towards the mother during labour $[9,12]$. Yet, by introducing simple but universal interventions improving care during delivery can become a reality. 
Evidence from the recent array of studies $[7,11,13]$ has it that many developing countries, Uganda inclusive, are yet to take a stride in the introduction and implementation of interventions and program against maternal abuse and neglect. However the evidence base of data required informing policy makers is still too weak due to paucity of studies on maternal abuse and neglect in this region. Despite increasing recognition of women's health needs in Uganda, little has been done to improve the way primary care physicians handle the pregnant women.

The forms of cultural and gender discrimination associated with patient abuse and neglect are copied or inherited by the health workers right from their communities[14] and training institutions [15] thus they are brought up in the culture and live in it. In the all, just as for other reproductive health issues, paucity of data exists on the influence of patient abuse on the utilization of skilled delivery services in Uganda. It is important, therefore, to understand the specific factors that perpetuate patient abuse in these rural Sub- Saharan African settings, since these may be considerably different from other settings found in the literature $[12,13$, 16]. Indeed, most the currently available date doesn't give statistically significant estimate of the magnitude of this problem. A lot of gaps still exist in the information about the health effects of maternal abuse and its relation with the health seeking behaviors of the mother. The paucity of the linkage between patient abuse and neglect the choice of the delivery service by the patient and the outcomes of the delivery there after, offered the basis for this study.

\section{Methods}

\section{Study settings}

The study was conducted in Bushenyi district in S.W Uganda with an estimated population of 487,432 [14]. The district has 29 health units, 23 of which (79\%) are public health facilities. There are three general hospitals which provide referral services and comprehensive Emergency Obstetric Care (EmOC). Two health centre IVs which provide basic EmOC and nine health's centre IIIs which provide delivery services but no EmOC. The rest are health centers with no delivery services. Although $70 \%$ of the population lives within $5 \mathrm{~km}$ radius of a health unit in Bushenyi, only one-third of these health units provide institutional deliveries and other reproductive health services. Most of the population in this district is rural (75\%), living on substance agriculture and cattle keeping as the main economic activity. Bushenyi has a high proportion of women attending antenatal care (ANC) at about 91\% [17]. However the district has one of the lowest proportions of women delivering under skilled attendance at $52 \%[14]$.

\section{Sampling}

In this cross sectional study a sample size of 374 respondents, determined by Wayne (1987) formula for infinite population proportions was be used. All the health facilities with delivery services in the district were included in the study. The respondents were targeted for interview before they left the health facilities after discharge. Since the health facilities deliver a few patients per day (0-5 patients) all patients discharged from the health facilities following delivery were included in the sample. Of all the eligible women identified for the interviews only $4(1.5 \%)$ refused to participate citing exhaustion and lack of time as the reasons for refusal.

\section{Data collection}

A pretested structured questionnaire was used to collect data on socio-demographic factors including demographic characteristics, history of abuse or neglect during the delivery, form of abuse, and the circumstance under which the abuse occurred. Research assistants trained on the data collection procedures carried out interviews while one of the principal investigators (AK) played a supervisory role. The data collected included basic personal demographic data of the respondents, basic obstetric history, and perception about the delivery service received during the current delivery and instances of abuse, or neglect during the delivery. 


\section{Data analysis}

The questionnaires were checked for completeness in the field. The data was entered and analysed using SPSS soft ware version 17. Descriptive statistics were used to analyse the socio-demographic variables of the participants and the prevalence of patient abuse which was expressed in terms of the proportion of the patient abuse during child birth in the health facilities. The outcome variable was patient abuse during childbirth. A two-sided $P$-value $<$ \pm 0.05 was considered statistically significant. Crude Odds ratios (COR) with $95 \%$ confidence intervals (CI) were used to assess for association during bivariate analysis. All those factors that were found to be significantly associated with patient abuse from bivariate analysis were considered for logistic regression analysis to generate a model to explain patient abuse during childbirth in the health facility. In logistic regression analysis, the backward likelihood ratio method was used to test the model and each of the predictors was assessed for the percent of variance it explained in the model.

\section{Ethical issues}

This study was approved by Texila American University School of Public Health and Kampala International University Research and ethics Committee. All the patients who participated gave verbal consent to the interviews.

\section{Results}

This study provides a basis for understanding patient abuse and neglect by health workers during child birth in south western Uganda. In the study, we had two working specific objective; to establish the magnitude of patient abuse and to assess the types of abuse commonly inflicted upon the patients delivering in health facilities by health workers in South Western Uganda. Data was collected from 372 respondents using interviewer administered questionnaires. We distributed a total of 376 questionnaires and received back 372 properly filled questionnaires, giving a response rate of $98.9 \%$.

\section{Study participants}

The data presented here is the result of analysis of the 372 questionnaires $(n=372)$ uniquely completed in the survey among women who had just delivered in the health facilities at the time of discharge. Of the 372 respondents, $101(27.0 \%)$ had delivered for the first time (parity 1) $241(64.9 \%)$ had parity $2-4$ and $30(8.1 \%)$ had the parity 5 and above. About the age of the respondents 181 were in the age group 18- 25 years 121 were $26-35$ years and 70 were above 36 and above years. About the facilities they had delivered from 234 (62.2\%) had attended PNFP health facility and $138(37.8 \%)$ had attended public facilities. Again $58(16.2 \%)$ had delivered from level III health facilities, $148(40.5 \%)$ had delivered from level IV health facilities and 168 (43.3\%) had delivered from level V health facilities.

The prevalence and risk factors for patient abuse during childbirth: Overall, the prevalence of patient abuse among the respondents was found to be $41.1 \%(\mathrm{P}=0.013)$. However only $29.4 \%(\mathrm{p}=0.041)$ of the patients interviewed could report the incidences of abuse first time. In the rest (11.7\%), abuse incidences were uncovered after a deep inquiry. Almost all types including physical abuse, verbal abuse, patient neglect, non consensual treatment and financial extortion were mentioned in this study.

Table1 shows adjusted Odds Ratio (OR) with 95\% Confidence Intervals (CI) of the association between patient abuse during child birth with a number of factors including; the age of the patient, the parity of the mother and the level of facility they attended. 
South American Journal of Public Health

Volume 4, Issue 2, 2016

Table 1: The association (OR, 95\% CI) between age, parity, health facility level, health facility type and patient abuse during childbirth. Result of multivariate logistic regression analysis

Patient characteristics

$\mathbf{n}=372$

\begin{tabular}{|c|c|c|c|}
\hline & COR (95\% CI) & AOR (95\% CI) & p-value \\
\hline \multicolumn{4}{|l|}{ Age group (years) } \\
\hline $18-25$ & $1.73(1.02-2.92)$ & $1.95(1.47-2.60)$ & 0.00 \\
\hline $26-35$ & $0.72(0.72-1.23)$ & $0.52(0.32-0.56)$ & 0.06 \\
\hline$\geq 36$ & $1 \mathrm{ref}$ & 1 ref & \\
\hline \multicolumn{4}{|l|}{ Parity } \\
\hline 1 & $2.22(1.06-1.33)$ & $2.07(0.09-1.51)$ & 0.00 \\
\hline $2-4$ & $0.19(0.51-1.01$ & $0.50(0.31-1.35)$ & 0.04 \\
\hline$\geq 5$ & 1 ref & 1 ref & \\
\hline \multicolumn{4}{|l|}{ Facility level } \\
\hline Level III & $1.91(0.02-1.20)$ & $1.68(0.72-1.45)$ & 0.01 \\
\hline Level IV & $0.02(1.43-2.11)$ & $0.52(0.49-0.81)$ & 0.02 \\
\hline Level V (hospital) & 1 ref & 1 ref & \\
\hline \multicolumn{4}{|l|}{ Facility type } \\
\hline Public & $1.51(1.31-1.72)$ & $1.91(0.63-1.41)$ & 0.00 \\
\hline PNFP & $1 \mathrm{ref}$ & $1 \mathrm{ref}$ & \\
\hline
\end{tabular}

There was statistical significant association between the patient being in the age group 1525years (AOR 1.95, 95\% CI 1.47-2.60), having a parity of one (AOR 1.24, 95\% CI 0.09 0.512 ) and having delivered from a Health center III (AOR 1.68, 95\% CI 0.72-1.45). Also having no relative or friend around had statistically significant association with patient abuse (AOR 2.93, 95\% CI 0.47-2.90).

However a significantly lower association was seen between patient abuse and women parity of 2-4 (AOR $0.50,95 \%$ CI $0.31-1.35$ ), women in the group 26-35 (AOR 0.698, 95\% CI 0.51-0.95). Also delivering in a higher facility level (level IV and V) showed low statistical association with patient abuse.

Graph I: Shows the effect of patient abuse on patient satisfaction with the services received, their ability to seek the same service again and the ability to recommend the service to a friend. In general, of all the patients who reported dissatisfaction with the services received (241), $171(74.1 \%, \mathrm{p}=0.00)$ had been abused in one way or another as compared to $70(25.9 \%, \mathrm{p}=0.04)$ who reported satisfaction with the services received. Of all the patients who reported that they can never seek the same service again (117), $93(79.6 \%, p=0.03)$ had been abused as compared to $24(16.8 \%, \mathrm{p}=0.01)$ among those who reported ability to seek the same service.

Graph I: Effect of patient abuse on patient satisfaction, seeking the same service again and the ability to recommend the service to a friend.

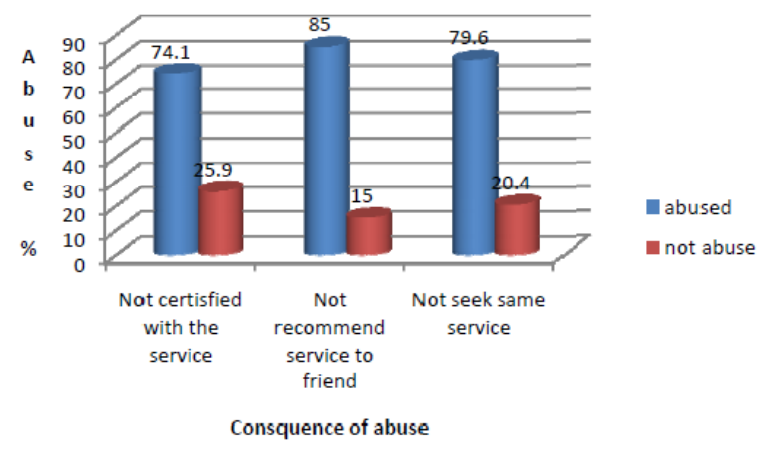


Types of abuse inflicted on women during childbirth: As seen in table II below, the commonest form of abuse reported was patient neglect $31.9 \%(p=0.000)$ where the patients were abandoned and left without the assistance of the health works when they needed it. The second most common form of abuse was verbal abuse reported by $29.3 \%$ of the respondents $(\mathrm{p}=0.001)$. This included shouting at the patient, scolding and using unnecessarily strong language.

Table II: Types of abuse experience by the patients in percentage, adjusted Odds Ratio (AOR) and associated $\mathrm{p}$-values $(\mathrm{n}=372)$

\begin{tabular}{llll} 
Abuse (type) & No. & \% & P-value \\
\hline Physical abuse & 73 & 19.7 & 0.032 \\
Verbal abuse & 109 & 29.3 & 0.001 \\
Patient neglect & 118 & 31.9 & 0.000 \\
Non consensual treatment & 51 & 13.8 & 0.935 \\
Denial of pain relief & 50 & 13.5 & 0.063 \\
Asked to pay bribe & 56 & 15.1 & 0.021
\end{tabular}

A significant number of the patients surveyed reported having been charged none official fees by the health workers $15.1 \%,(p=0.021)$ before any services could be provided to them. The amount charged depended on the discretion of the health worker but it ranged from Ugandan shilling equivalent of 2 US dollars to as much as 40 US dollars if the mother needed operative delivery.

Denial of pain relief was experience by $13.5 \%(p=0.063)$ of the patients. Physical abuse in the form of boxing, slapping, caning, pinching pushing was experienced by $19.7 \%(p=0.032)$ of the patients delivering in the surveyed health facilities. And non consensual treatment reported by $13.8 \%(\mathrm{p}=0.935)$. None of the respondents reported any incidence of sexual abuse in this study.

\section{Discussion}

The results of this study indicate a high prevalence of patient abuse during childbirth in south-western Uganda where it is significantly associated with patient factors as well as health facility factor. This study was part of a larger project of four studies aimed at establishing ways of eliminating patient abuse and improving patient care during childbirth in South Western Uganda. In the long run the study will contribute towards improving the uptake of supervised delivery services in the health facilities in Uganda with the ultimate the reduction of maternal and neonatal mortality and morbidity basing on the well known fact that health workers behavior towards patients is a key ingredient that shape the picture of the health system in the eyes of the service seekers. The studies are designed to provide information, which could be used by policy makers and implementers to design interventions to reduce patient abuse in the maternity setting. The results may not be generalized to the entire country but they provide valid insights into the prevalence of patient abuse during child birth, the forms of abuse experienced by the patients and the factors associated with the abuse that can be extrapolated to other parts of the country where such data is missing.

Inappropriate patient handling was identified as a possible cause for the failure to seek supervised delivery services or a delay in taking a decision to seek care resulting into complications of child birth. The study further shows a strong association between patient abuse and the decision making on location of birth place, the ability of the patient to ever come back to the health facility for the services and ability to recommend the services to friends. Moreover it has been reported that only $32 \%$ of women in this delivered in health care facilities [14]. 
South American Journal of Public Health

Volume 4, Issue 2, 2016

\section{Prevalence of patient abuse during childbirth}

The prevalence of patient abuse of $41.1 \%$ observed in our study is high compared to what other studies $[4,12,13]$ which have reported prevalence rates of between $9.1 \%-27 \%$. The explanation for this difference can be viewed in terms of the settings in which the study was conducted. Most of the studies we found in literature that tried to measure the prevalence of patient abuse were conducted in American and European settings, quite different from what is expected of an African setting. Unfortunately we did not come across any comparative study in the African setting that tried to measure the magnitude of patient abuse. In this study women across all spheres including rural and semi-urban residents expressed history of having been abused to some degree. This finding was divergent from Mvo et al (2005) who described abuse among the rural residents in South Africa as compared to the urban dwellers [6]. They attributed the difference to the appearance and the kemptinace of the patients as they reported to hospital which was dissimilar in the two groups of patients.

The young patients, age group 15-25 years reported more incidences of having been abused. This is a very unfortunate finding as this particular age group is comprised of very sensitive people who need to be handled with at most care. More over they are more prone to birth related complications [17] therefore scaring them away from receiving supervised delivery is time bomb for any health system. Maine (2005) reported similar finding among women southern Malawi [18]. They described adolescent patient being scolded for coming to a contraceptive clinic and most times being denied explanation of how to use the medicines given to them. The lower health facilities and the public health facilities were more commonly associated with patient abuse. In the context of this study, this could be a factor lack of close supervision of the health worker in the facilities where they are left on their own with very little or no supervision. The 'lonely feeling of out of place' may lead the health work to doing anything to their patients, probably out of will but can also be out of frustration. In fact most of the health facilities surveyed have lower level health workers with little experience and lower motivation levels.

In all, it was often difficult for the respondents to identify what abuse was and whether they have been abused or not. It often took the interviewer time to make deep inquiries in order to uncover the incidences of patient abuse. As Kyamuhendo (2010) pointed out, there is no 'clear cut' single dependable yard stick for identification of patient abuse [11]. Apparently in the rural African context, in which this study was conducted, most times patients take certain abusive actions of the health worker as normal to the health system. Depending on the existing situation, patients have to get satisfied with any form of treatment so long as they obtain positive results "a healthy baby". To them the end justifies the means, abused or not. As asserted by Lehmann (2013) most of the patients in the low-income countries hardly know their rights especially when it comes to seeking health care services [19].

Given such a high magnitude of abusive behaviors and mistreatment exhibited to patients seeking supervised delivery in the health facilities, it would be inappropriate to expect the proportion of pregnant mothers seeking supervised to increase. The marked disparity in prevalence of skilled supervised delivery the developed countries (up to $100 \%$ in UK and Canada) and the low income countries (37\% in Uganda) is partly attributable to the way patients are handled in the health facilities [14, 20 ]. Musisi et al. (2008) study found that lack of compassionate treatment was among the most common barriers to health facility deliveries that drive mothers to give birth in their communities [21]. In this study a significant proportion of patients $(33.7 \%)$ made it categorically clear that they can never come back to deliver in the same health system and $36.1 \%$ would never refer friends to such an abusive health system. The implication of this is that the pregnant women are left with very little choices other than delivering under unsupervised condition in their communities. According Kabakyenga (2012) women in low-income countries have several options for location of childbirth depending on their perception on how they will be treated including home delivery, which may be solitary or assisted by unskilled assistants, and health facility delivery which can either be in a public or private institution [17]. Neema (2002) in her study conducted the 
same region (south-western Uganda) also reported that women find themselves in the dilemma regarding the choice for location of birth as they are torn between the dangers of delivering at home and the disrespectful care in the healthcare facility[22]. The results from our study indicate that women who, solitarily, reported to the health facility to give birth are more likely to be abused by the birth attendants than those who were accompanied by others (partner, friends, and relatives). This finding supports the results from study Pombe (2010) where women reported preference for home/solitary confinement during childbirth if they had no one to accompany them to the health facility in order to protect their own integrity [23]. Moreover, in low-income countries home births are inherently risky, should a complication such as prolonged labour occur then a possibility of adverse outcome is highly likely [14].

In Ghana, Kuffo et al. (2009) found that poor quality of care and bizarre labour suit experiences adversely affect the reputation of the public health care system with the resultant high risk community childbirths [9]. The assessment of the quality of maternity services received by the patients in relation to patient abuse in the labour suite was the focus of a study by Thaddeus (2004) Consistent with the current study, they found that the attitude of the midwives towards patients, availability of delivery equipment and supplies were most influential in the choice of where a mother goes for delivery [24]. Improving these and related parameters would lead to a consequential raise of facility delivery by $55-75 \%$, which would overturn maternal mortality in this region by over $50 \%[2,25]$.

\section{Types of abuse}

The result from our study indicate that there are various types of abuse experienced by the women in this region included verbal abuse, discrimination, abandonment, non consensual treatment, financial extortion and physical abuse. Similar forms were reported by Shones et al. (2005) in their study conducted in Morogoro Tanzania [20]. However in their study incidence of physical abuse was reported to be very scarce.

Physical abuse: In the current study, physical abuse taking the form of boxing, slapping, pinching, pushing and forced treatment or procedures emerged strongly with some patients showing signs such as bruises and swellings at the time of interview. This shows the extremes of the forms of mistreatment occurred in this community. Von (2000) described how women would get hit or beaten during labor if they yelled too much or "talked back" to the midwives [26]. They reported that this behavior was witnessed by many of their responds being inflicted to others patients. Other physical actions such as being forced to deliver while lying down with the knees pulled "up", and other uncomfortable and frightening positions were also commonly reported. Similar to other dimensions of abuse, physical abuse is often inflicted in the instance of a women expressing their preferences as opposed to a "modern" mold have been reported $[1,2,27,28]$.

Verbal abuse: Mvo et al. found verbal abuse in the form of criticism levied against the delivering mothers to be very common [6]. They found that the verbal abuse entailed outright shouting or harsh remarks towards the patient. According to their finding, verbal abuse was discriminatory in nature, in that the women who were not following the "rules" or were not presenting themselves as "modern women" were more likely to be berated. In the current study we found this form of abuse to be statistically significantly related to the 'young' age of the patient and the parity of the patient involved.

Zhang et al. (2011) reported patients being scolded for not pushing hard enough during the second stage of labour. Similar reports were given many respondents in the current study [27]. Although this action is intended to encourage the mother to put in more effort and push harder for the baby to be delivered faster, it doesn't call for such extreme degrees of verbal flexion that is tantamount to patient abuse. The midwives are expected to have learnt better techniques of encouraging the woman to push out the baby. Similar to our findings, Jewkes et al. (2005) described more common criticisms against the patients in labour including; critiques of a woman's economic status such as wearing old or dirty clothes, critiques of her use of traditional medications and her history of antenatal attendance [3]. 
Feeling ignored or neglected: Mwesigye (2008) described how women expressed fear of arriving at a facility and being ignored or delivering without the assistance of the midwife [27]. They described an occasion during a night delivery, where the midwife kept at her home within the health facility, but was unwilling or unable to come and deliver the mother in the labour suit.

Goergen et al. (2004) narrated how a group of health providers were in their nurses' room conversing and taking tea while a mother in labour was yelling helplessly in the labour suit and yet none of them was willing to come to her rescue [10]. In Malawi a mother almost lost her baby when she fell out without the attention of the midwife [29]. The baby was save when the nurse ran and caught the baby as she landed to the ground. In South African pregnant women admitted to the same ward were found assisting each other to deliver in the absence of a midwife $[29,30]$.

Soliciting of bribes from patients: D'Oliveira et al. (2005) described situations where mothers were expected to give material items to the midwives in the facilities before they re assisted to delivery [28]. In some situations, they said, the women were asked to pay a "thank you" to providers following a delivery. This of course was none official and against the will of the patient. Eastley (2007) found that women who had delivered out of hospital in their previous deliveries were asked to pay a fine for home delivery before being attended to [13].

Several papers have reported in official charges level to patients inform of bribes (referred to as a 'kick') especially in public facilities where payment was abolished in this country [30]. In very busy hospitals where overcrowding and floor cases are the order of the day, patients are charged none official fees for the beds or else they stay among the floor cases [31]. Most times it's not because the health workers are under remunerated but more of a moral issue drives corruption among health workers. Moreover they ask for bribes from rural poor women well knowing how deeply they entrapped in poverty and insufficient social amenities. Some of the health workers are well to do persons only craving for undue happiness and wanting to be classy. The perpetual habit is driven by extravagancy and expenditure beyond their means to ripping where they never saw. Corruption has been described to be deeply entrenched into the moral fabrics of the health system [32].

Discriminatory treatment: Shanon et al. (2005) described how certain sectors of women could more easily access supplies or services at facilities than others [20]. They found that women of a higher social status, those who knew someone working within the facility or were somehow, for unknown reasons, favored by providers. In their study they described how nurses could discriminatorily decide - "upon seeing a woman coming to the facility or not" "whether they would provide prompt services to her or not. This form of retrogressive behaviors' such as basing on religion or tribes in service delivery, a form of persistent apartheid, was found to be rare in this region.

Other studies including $[31,32]$ have attached the discrimination of mothers to their failure to pay the bribe, dirtiness, poorly dressed up or a look like rural women. Also mothers are discriminated according to their political and social background [31].

Recommendation for future research: In-depth studies to investigate into the reasons why health workers abuse their patients and the circumstance under which the abuses occur need to be undertaken. Furthermore an extensive quantitative study at a national scale is needed to uncover regional differences in the extent of abusive patient care.

\section{Conclusions and recommendations}

To achieve a reduction of maternal morbidity and mortality must start with a consented effort to reduce the proportion of women delivering in the communities without skilled attendance. A conducive delivery environment can only be achieved with compassionate nursing care to all mothers seeking the care of health workers. Patient abuse of any form is detrimental as lenders the health system unpopular to the communities and reduces service utilization with adverse consequences to the patients. 
Close supervision midwives while caring for the patients in labour is essential to reduce the incidences of patient abuse as well as keeping the company of the relatives or friends to the patients around the labour suite. Community mobilisation and empowerment through health education using the existing structure like the Community Health Workers to ensure awareness of their fundamental rights is highly recommended. Capacity building through human right sensitization for both patients and health workers is vital at all facilities levels. The patients should be made aware of where to seek redress in case of abuse. A multi-sectoral approach to improve the literacy of the women poverty reduction is essential to reduce gender based violence against the pregnant women which is central in improving the uptake of maternal services.

\section{References}

[1] World Health Organisation. The Conceptual Framework for the International Classification for Patient Safety. Geneva: The World Health Organisation; Social Q (2009) 23:333-343.

[2] UNICEF. The State of the World's Children 2013. New York, NY: United Nations Publications; (2013) 13: 17

[3] Jewkes R, Abrahams N, Mvo Z. Why do nurses abuse patients? Reflections from South African obstetric services. Soc Sci Med (2005) 47:1781-1795.

[4] Filippi V, Ronsmans C, Campbell OM, Graham WJ, Mills A, Borghi J, Koblinsky M, Osrin D. Maternal health in poor countries: the broader context and a call for action. Lancet (2006) 368:15351541.

[5] Drakles, Bowser D, Hill K (2010) Exploring evidence for disrespect and abuse in facility-based childbirth. USAID-TRAction Project, Harvard School of Public Health (2010) 2: 36

[6] Mvo Z Abrahams N. Why do nurses abuse patients? Reflections from South African obstetric services. Soc Sci Med (2005) 47:1781-1795.

[7] Isola A, Backman K, Voutilainen P, Rautsiala T. Family members' experiences of the quality of geriatric care. Scand J Caring Sci (2003) 17:399-408.

[8] Schraiber LB, Diniz SG. Violence against women in health-care institutions: an emerging problem. Lancet (2005) 359:1681-1685.

[9] Kuffo SE, Mbaruku G, McCord CW, Moran M, Rockers PC, Galea S. Bypassing primary care facilities for childbirth: a population-based study in rural Ghana. Health Policy Plan (2009) 24:279288.

[10] Goergen T: A multi-method study on elder abuse and neglect in nursing homes. The Journal of Adult Protection 2004, 6:15-25.

[11] Kyamuhendo L, Abbey M. Please understand when I cry out in pain: women's accounts of maternity services during labour and delivery in Ghana. BMC Public Health (2005) 5:140.

[12] Zhang Z, Schiamberg L, Oehmke J, Barboza G, Griffore R, Post L, Weatherill R, Mastin T. Neglect of older adults in Michigan Nursing Homes. J Elder Abuse Negl (2011) 23:58-74.

[13] Eastley, R. and G.K Wilcock. "Prevalence and correlates of Aggressive Behaviors Occurring in Patients with Alzheimer's Disease." International Journal of Geriatric Psychiatry (1997) 12: 484-487.

[14] Kabakyenga JK, Ostergren PO, Turyakira E, Mukasa PK, Odberg Pettersson K. Individual and health facility factors and the risk for obstructed labour and its adverse outcomes in south-western Uganda. BMC Pregnancy Childbirth (2011) 11:73.

[15] Chirwa E, Odland JØ, Bjune G. Do Malawian women critically assess the quality of care? A qualitative study on women's perceptions of perinatal care at a district hospital in Malawi. Reprod Health (2012) 9:30

[16] Entwistle V, McCaughan D, Watt I, Birks Y, Hall J, Peat M, Williams B, Wright J: Speaking up about safety concerns: multi-setting qualitative study of patients' views and experiences. Qual Saf Health Care (2010) 19:1-7.

[17] Kabakyenga JK, Ostergren PO, Emmelin M, Kyomuhendo P, Odberg Petterss on K. The pathway of obstructed labour as perceived by community members in south western Uganda: a grounded theory study. Global Health Action (2012) 4:8529. 
South American Journal of Public Health

Volume 4, Issue 2, 2016

[18] Maine D, Rosenfield A. Maternal mortality-a neglected tragedy. Where is the M in MCH? Lancet (2005) 2:83-85.

[19] Lehmann, L.S., R.A McCormick, and K.W Kizer. “A Survey of Assaultive Behavior in Veterans Health Administration Facilities. "Psychiatric Services (1999) 50: 384-389.

[20] Shanon JH, Baer HA, Singer M. Toward a critical medical anthropology. Soc Sci Med (2005) 23:95-98.

[21] Musisi B, Berry JM. Who's judging the quality of care? Indigenous Maya and the problem of "not being attended". Med Anthropol (2008) 27:164-189.

[22] Neema S. Why child birth has become a night mare: a population-based study in rural Uganda. Health Policy Plan (2004) 24:279-288.

[23] Pombe W. The other side of caring: abuse in a South African maternity ward. J Reprod Infant Psychol (2010) 28:84-101.

[24] Thaddeus S, Maine D. Too far to walk: maternal mortality in context. Soc Sci Med (1994) 38:1091-1110.

[25] Reichenheim ME, Zylbersztajn F, Moraes CL, Lobato G. Severe acute obstetric morbidity (nearmiss): a review of the relative use of its diagnostic indicators. Arch Gynecol Obstet (2009) 280:337343.

[26] Von Dietze E, Orb A. Compassionate care: a moral dimension of nursing. Nurs Inq (2000) 7:166174.

[27] Mwesigye H. Who's judging the quality of care? Indigenous Maya and the problem of "not being attended". Med Anthropol (2008) 27:164-189.

[28] d'Oliveira AFPL, Diniz SG, Schraiber LB. Violence against women in health-care institutions: an emerging problem. Lancet (2005) 359:1681-1685.

[29] Aborigo RA, Hodgson A, Engmann CM. 'They treat you like you are not a human being': maltreatment during labour and delivery in rural northern Uganda. Midwifery (2014) 30 (2):262-268.

[30] Penney LM, Spector PE. Job stress, incivility, and counterproductive work behavior (CWB): the moderating role of negative affectivity. J Organ Behav (2005) 26:777-796

[31] Bowser D, Hill K. Exploring evidence for disrespect and abuse in facility-based childbirth. USAID-TRAction Project, Harvard School of Public Health (2010) 21: 64-69.

[32] Fonn S, Xaba M. Health Workers for Change: A manual to improve quality of care. Geneva, Switzerland: World Health Organization, Special Program for Research and Training in Tropical Diseases; (1995) 7: 64- 89. 